\title{
The Role of Conduction Delay in Creating Sensitivity to Interaural Time Differences
}

\author{
Catherine Carr, Go Ashida, Hermann Wagner, Thomas McColgan \\ and Richard Kempter
}

\begin{abstract}
Axons from the nucleus magnocellularis (NM) and their targets in nucleus laminaris (NL) form the circuit responsible for encoding interaural time difference (ITD). In barn owls, NL receives bilateral inputs from NM, such that axons from the ipsilateral NM enter NL dorsally, while contralateral axons enter from the ventral side. These afferents act as delay lines to create maps of ITD in NL. Since delay-line inputs are characterized by a precise latency to auditory stimulation, but the postsynaptic coincidence detectors respond to ongoing phase difference, we asked whether the latencies of a local group of axons were identical, or varied by multiples of the inverse of the frequency they respond to, i.e., to multiples of $2 \pi$ phase. Intracellular recordings from NM axons were used to measure delay-line latencies in NL. Systematic shifts in conduction delay within NL accounted for the maps of ITD, but recorded latencies of individual inputs at nearby locations could vary by $2 \pi$ or $4 \pi$. Therefore microsecond precision is achieved through sensitivity to phase delays, rather than absolute latencies. We propose that the auditory system "coarsely" matches ipsilateral and contralateral latencies using physical delay lines,
\end{abstract}

\footnotetext{
C. Carr $(\bowtie)$

Department of Biology, University of Maryland, College Park, MD, USA

e-mail: cecarr@umd.edu

G. Ashida

Cluster of Excellence "Hearing4all”, University of Oldenburg, Oldenburg, Germany

e-mail: go.ashida@uni-oldenburg.de

H. Wagner

Institute for Biology II, RWTH Aachen, Aachen, Germany

e-mail: wagner@bio2.rwth-aachen.de

T. McColgan $\cdot$ R. Kempter

Institute for Theoretical Biology, Department of Biology,

Humboldt-Universität zu Berlin, Berlin, Germany

e-mail: thomas.mccolgan@gmail.com

R. Kempter

e-mail: r.kempter@biologie.hu-berlin.de

(C) The Author(s) 2016

P. van Dijk et al. (eds.), Physiology, Psychoacoustics and Cognition in Normal and Impaired Hearing, Advances in Experimental Medicine and Biology 894, DOI 10.1007/978-3-319-25474-6_20
} 
so that inputs arrive at NL at about the same time, and then "finely" matches latency modulo $2 \pi$ to achieve microsecond ITD precision.

Keywords Coding $\cdot$ Interaural time difference $\cdot$ Plasticity $\cdot$ Models $\cdot$ Rate

\section{Introduction}

A key feature of ITD sensitivity is how the observed microsecond delays may be created by neural elements that are both noisy and slow. There are two possibilities, illustrated by the example of a coincidence detector neuron that responds to a pure sine tone, presented to both ears at an ITD of $0 \mu \mathrm{s}$, or sound in front of the animal. One strategy requires equal conduction latencies from each ear to the coincidence detector, such that the inputs would arrive with equal latency and equal phase. The second strategy relies on relative delays and would simply require that inputs arrive at the same phase. Due to the periodicity of the sine wave, both scenarios would produce the same output. This coding strategy remains viable for non-periodic sounds because the tonotopic organization of the auditory system generates ringing properties of the inputs characteristic of the particular frequency (Carney et al. 1999; Recio et al. 1998; Wagner et al. 2005). Previously we had shown that the inputs from the cochlear nuclei act as delay line inputs to form maps of ITD in the barn owl (Carr and Konishi 1990), but could not determine whether the delay lines had identical conduction latencies or were matched for phase only.

To differentiate between the two hypotheses, we combined measurements of the extracellular potential or neurophonic with intracellular measures of click latency in NL. For mapping the ITD we used the neurophonic, because it reflects local ITD sensitivity (Sullivan and Konishi 1986; Kuokkanen et al. 2010; Mc Laughlin et al. 2010; Kuokkanen et al. 2013), and to measure delay we used clicks, because their temporal occurrence is precise to within about $20 \mu \mathrm{s}$ (Wagner et al. 2005). We found the microsecond precision needed to construct maps of ITD is achieved through precisely regulated phase delays, rather than by regulation of absolute latency, consistent with the observation that nucleus laminaris neurons respond over a wide range of integer multiples of their preferred interaural phase difference (Christianson and Peña 2006). Thus latencies from the ear to the point of coincidence detection need only be adjusted within a stimulus period, creating a flexible yet precise system for detection of sound source sound location. This high precision approach should also benefit other binaural properties of the auditory system, such as resistance to background noise and reverberation. 


\section{Methods}

The experiments were conducted at the University Maryland. Four barn owls (Tyto alba) were used to collect these data, and procedures conformed to NIH guidelines for Animal Research and were approved by the Animal Care and Use Committee of the Universities of Maryland. Anaesthesia was induced by intramuscular injections of $10-20 \mathrm{mg} / \mathrm{kg}$ ketamine hydrochloride and 3-4 mg/kg xylazine, with supplementary doses to maintain a suitable plane of anaesthesia. Recordings were made in a sound-attenuating chamber (IAC, New York). Intracellular recordings were amplified by an Axoclamp 2B, and then by a spike conditioner (PC1, Tucker-Davis Technologies (TDT), Gainesville, FL). Acoustic stimuli were digitally generated by custom-made software ("Xdphys" written in Dr. M. Konishi's lab at Caltech) driving a signal-processing board (DSP2 (TDT)). Acoustic signals were fed to earphones, and inserted into the owl's left and right ear canals, respectively. At a given recording site, we measured frequency tuning, then tuning to ITD, and responses to monaural clicks.

\section{Results}

Axons from NM form presynaptic maps of ITD in NL. These inputs generate a field potential that varies systematically with recording position and can be used to map ITDs. In the barn owl, the representation of best ITD shifts systematically in NL, forming multiple, largely overlapping maps of ITD (Fig. 1a). We had previously found that conduction delays could account for the shift in maps of ITD (Carr et al. 2013).
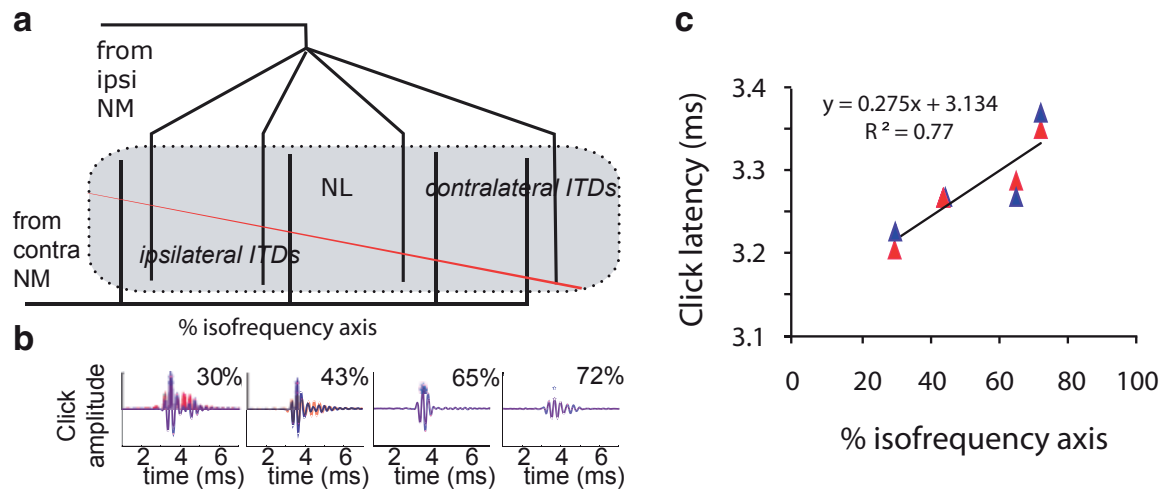

Fig. 1 a Schematic outline of an isofrequency slab in NL showing delay-line inputs from each side, with the " $0 \mu$ s-ITD line" in red. $\mathbf{b}$ Click responses were recorded at best ITDs around $0 \mu \mathrm{s}$, at four different locations along a $3.6 \mathrm{kHz}$ iso-frequency slab in NL. Superimposed ipsilateral (blue) and contralateral (red) averages of 128 click responses from recording sites at 30, 43, 65 and $72 \%$ along the isofrequency axis, measured from 3-D reconstructions of NL with lesions at each recording location. c Click delays from b. showed a systematic increase in latency with mediolateral position in NL for both ipsi- and contralateral responses 


\subsection{Measuring Latency with Click Delays}

Our mapping studies showed that the representation of frontal space ( $0 \mu \mathrm{s}$ ITD) in NL shifted systematically to more ventral locations with the progression from medial to lateral along each tonotopic slab (red line, Fig. 1a, see Carr et al. 2013). To determine if changes in conduction velocity formed the basis of the systematic shift, we used click delay to measure latencies at different mediolateral positions. Measurements of click latency provided reliable measures of conduction delay (Köppl and Carr 2008; Wagner et al. 2005), because click stimuli were temporally precise. Nevertheless, click stimuli evoked an oscillatory response (Ruggero et al. 1986; Wagner et al. 2005; Wagner et al. 2009), in which typically several peaks and troughs could be distinguished (Fig. 1b).

Neurophonic responses to ipsi- and contralateral clicks at best ITDs at or near $0 \mu$ senerated similar ipsi- and contralateral click responses, which largely overlapped (Fig. 1b). Responses to both ipsilateral and contralateral stimulation showed a steady increase in latency along the iso-frequency axis in NL (Fig. 1c), i.e., systematic changes in conduction delay underlay the formation of the maps of ITD. To determine whether the delay lines had identical conduction latencies or were matched for phase only, we compared neurophonic multiunit click recordings with intracellular recordings from individual NM axons in NL.

\subsection{Latencies of Adjacent Recordings Can Vary by Multiples of $2 \pi$}

Intracellular recordings from NM axons in NL supported the hypothesis that oscillatory neurophonic click responses were composed of many NM click responses. Averaging intracellular click recordings from NM afferents revealed two to five PSTH peaks (Fig. 2a). PSTH peaks were separated in time by intervals equal to the inverse of the neuron's best frequency (BF), a $2 \pi$ phase interval. Individual spike timing varied, consistent with phase locking (Anderson et al. 1970; Sachs et al. 1974; Joris et al. 1994; Köppl 1997). For each intracellular recording, we quantified the temporal distance between the PSTH peaks. All showed a similar $2 \pi$ separation of peaks. Adjacent recordings responded at very similar phases, suggesting adjacent axons fire in synchrony or at multiples of $2 \pi$ (Fig. $2 b$, note clustered adjacent symbols).

In the penetration through the dorsoventral dimension of $5.2 \mathrm{kHz}$ NL shown in Fig. 2b and 2c, recordings between 205-270 $\mu \mathrm{m}$ depths yielded three contralateral units with similar phase, but a latency difference of $4 \pi$ (Fig. 2c, black arrow). The first two recordings had latencies of 3.33 and $3.32 \mathrm{~ms}$, the third $2.91 \mathrm{~ms}$. Thus, the recording with the shorter latency occurred almost $4 \pi$ or $0.38 \mathrm{~ms}$ earlier than the first two, given a stimulus period of $192 \mu \mathrm{s}(3.33 \mathrm{~ms}-2 * 0.192 \mathrm{~ms}=2.94 \mathrm{~ms})$. In other words, the phase delay of these peaks exhibited a narrow scatter, while the signal-front delay showed a large scatter, as had also been observed for the neurophonic (Wagner et al. 2005). A similar measurement at $865 \mu \mathrm{m}$ had a latency of 

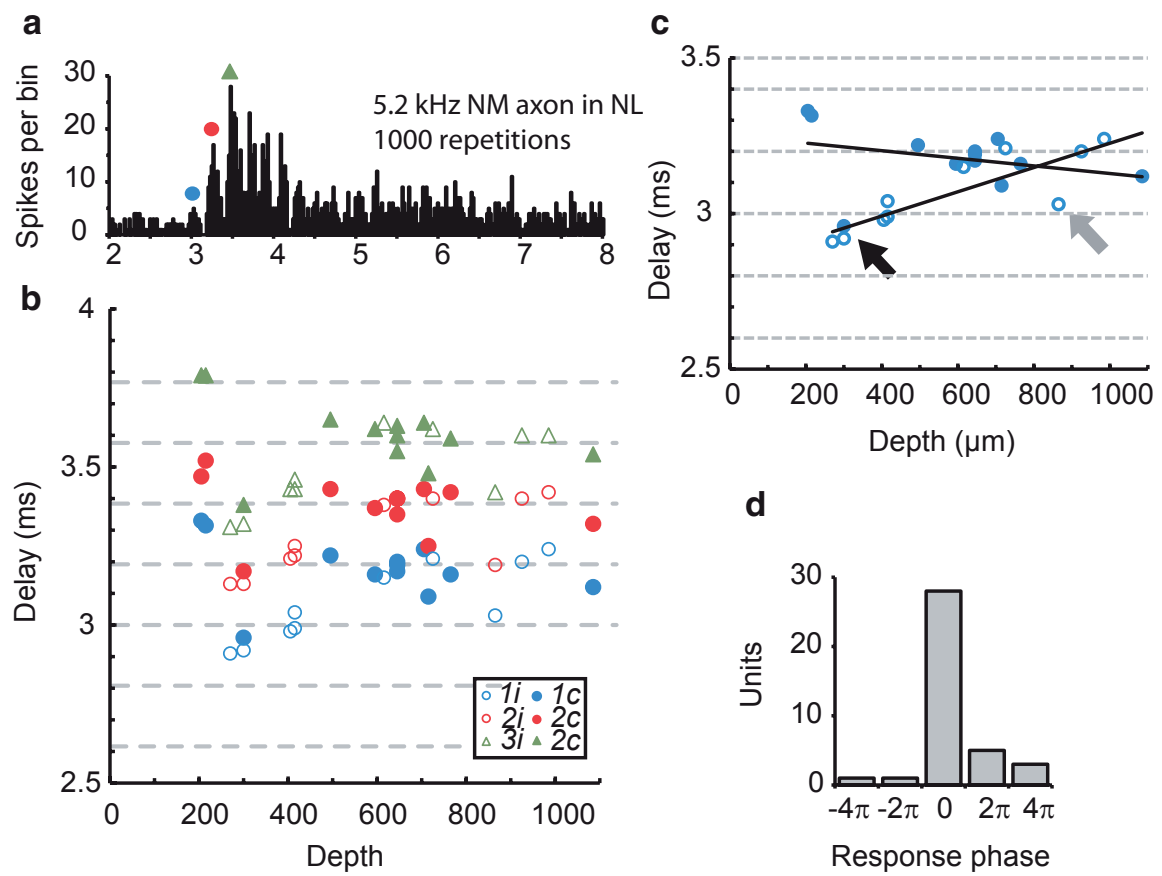

Fig. 2 a Delay of the first three PSTH peaks recorded in a single NM axon within NL. Coloured symbols mark peaks in the PSTH. Blue circle $=$ first peak, or signal-front delay, red circle $=$ second peak and green triangle $=$ third peak. $\mathbf{b}$ Click latencies in $22 \mathrm{NM}$ afferents recorded in a single penetration through the $5.2 \mathrm{kHz}$ region of left NL. Both ipsilateral (open symbols) and contralateral (closed symbols) responses were encountered, symbols as in a. The latencies of the first 3 PSTH peaks for these afferents varied by multiples of 1/BF, shown as horizontal dashed lines $192 \mu \mathrm{s}$ apart. The first three PSTH peaks for each recording were shown with respect to recording depth as a vertical array of blue circles (first peak), red circles (second peak, $+2 \pi$ ) and green triangles (third peak, $+4 \pi$ ). $\mathbf{c}$ Latencies of the ipsilateral NM axons increased with depth (open symbols, $\mathrm{y}=0.4 \mathrm{~s} / \mathrm{mx}+2.84 \mathrm{~ms}, \mathrm{r}^{2}=0.7$ ), while recordings from contralateral $\mathrm{NM}$ axons in NL decreased in latency with depth (closed symbols, $\mathrm{y}=-0.12 \mathrm{~s} / \mathrm{m} \mathrm{x}+3.25, \mathrm{r}^{2}=0.10$ ). d Most data from 3 penetrations through NL ( $n=38$ units, 2 owls) showed systematic shifts in latency with recording depth, consistent with models of delay (see $2 \mathrm{~b}$, lumped in $0 \mu \mathrm{s}$ bin). Other units were $2 \pi$ or $4 \pi$ or later than neighbouring units (c, arrows)

3.03, while the adjacent ipsilateral recordings at 725 and $925 \mu \mathrm{m}$ had latencies of 3.21 and $3.20 \mathrm{~ms}$ (Fig. 2c, grey arrow). Most measurements had similar phase and latency (Fig. 2d, bar marked 0 ), but some occurred $2 \pi$ earlier, and others $2 \pi$ or $4 \pi$ later than the rest of the population (Fig. 2d).

In general, changes in delay with depth were consistent with the NM axons acting as delay lines (Carr and Konishi 1990), and with modelled conduction velocities in McColgan et al. (2014). Ipsilateral recordings showed an increase in latency with recording depth in NL (open symbols in Fig. 2), while contralateral recordings (closed symbols) showed a decrease in latency with depth, consistent with the delay line geometry. The regression lines in Fig. 2c predicted velocities of +2.5 and $-8.3 \mathrm{~m} / \mathrm{s}$, consistent with modelling results (McColgan et al. 2014) and measure- 
ments from previous studies (Carr and Konishi 1990). Nevertheless, we point out that while these depth-dependent changes in delay would be expected from multiple recordings from the same NM axon, they were not required for ITD sensitivity. Sensitivity to ITDs should be instead conferred by the differences in phase.

Both extracellular neurophonic recordings and intracellular NM axonal recordings in NL revealed maps of ITD, created by precisely regulated conduction delays from intrinsically noisy elements. The microsecond precision needed to construct these maps of ITD appears to be achieved through precisely regulated phase delays, rather than by regulation of absolute latency.

\section{Discussion}

Delays from each side must be matched for coincidence detection to occur. How? Coincidence requires microsecond precision, which may be achieved by a two-step process, where latencies from each ear are matched during development, and then precisely matched modulo $2 \pi$. This was first proposed by Gerstner et al. (1996), who postulated that the necessary degree of coherence in the signal arrival times could be attained during development by an unsupervised Hebbian learning rule that selects connections with matching delays from a broad distribution of axons with random delays. Our data support this matching hypothesis, with coarse delays laid down through normal development, and precise, i.e. $\pm 20 \mu$ s modulo $2 \pi$, delays regulated by some as yet unknown activity dependent processes (Seidl et al. 2010).

Anatomical data are consistent with coarsely matched latencies. For each frequency, latencies in barn owl NM and in the midline cross fibre tract are similar, consistent with a common path from the ear into the brain (see Fig. 7 in Köppl 1997). Our previous measures of latency did not have the precision of the current intracellular recordings, with ipsilateral and contralateral delays at the dorsal and ventral borders of NL of $2.82 \pm 0.24$ and $2.87 \pm 0.21 \mathrm{~ms}$ respectively (Carr and Konishi 1990). Note the standard deviations of about $200 \mu \mathrm{s}$ in these early recordings of latency. Our current recordings support the hypothesis that within NL, latencies vary by multiples of $2 \pi$, and may be precisely regulated at a fine time scale, in order to create a cycle by cycle representation of the stimulus at the point(s) of coincidence detection (Funabiki et al. 2011; Ashida et al. 2013). Modulation by multiples of $2 \pi$ is also consistent with cross-correlation and spike timing dependent plasticity models (Gerstner et al. 1996; Kempter et al. 1998; Pena and Konishi 2000; Fischer et al. 2008). Variability in response latency also characterizes mammalian auditory nerve and cochlear nucleus recordings (Sanes and Constantine-Paton 1985; Carney and Yin 1988; Young et al. 1988).

Acknowledgments This research was sponsored by NIH DC00436 to CEC, by NIH P30 DC04664 to the University of Maryland Center for the Comparative and Evolutionary Biology of Hearing, by the German Research Foundation (DFG, Wa-606/12, Ke-788/1-3, 4) and the Bundesministerium für Bildung und Forschung (BMBF: 01GQ0972 and 01GQ1001A, Bernstein Collaboration Temporal Precision, 01GQ07101 to HW and 01GQ07102 to RK,), and by the cluster of Excellence, "Hearing4all" at the University of Oldenburg (GA). 
Open Access This chapter is distributed under the terms of the Creative Commons AttributionNoncommercial 2.5 License (http://creativecommons.org/licenses/by-nc/2.5/) which permits any noncommercial use, distribution, and reproduction in any medium, provided the original author(s) and source are credited.

The images or other third party material in this chapter are included in the work's Creative Commons license, unless indicated otherwise in the credit line; if such material is not included in the work's Creative Commons license and the respective action is not permitted by statutory regulation, users will need to obtain permission from the license holder to duplicate, adapt or reproduce the material.

\section{References}

Anderson DJ, Rose JE, Hind JE, Brugge J (1970) Temporal position of discharges in single auditory nerve fibers within the cycle of a sine-wave stimulus: frequency and intensity effects. J Acoust Soc Am 49(2):1131-1139

Ashida G, Funabiki K, Carr CE (2013) Biophysical basis of the sound analog membrane potential that underlies coincidence detection in the barn owl. Front Comput Neurosci 7:102. doi:10.3389/fncom.2013.00102

Carney L, McDuffy M, Shekhter I (1999) Frequency glides in the impulse responses of auditorynerve fibers. J Acoust Soc Am 105:2384

Carney LH, Yin TCT (1988) Temporal coding of resonances by low-frequency auditory nerve fibers: single-fiber responses and a population model. J Neurophysiol 60:1653-1677

Carr CE, Konishi M (1990) A circuit for detection of interaural time differences in the brain stem of the barn owl. J Neurosci 10(10):3227-3246

Carr CE, Shah S, Ashida G, McColgan T, Wagner H, Kuokkanen PT et al (2013) Maps of ITD in the nucleus laminaris of the barn owl. Adv Exp Med Biol 787:215-222. doi:10.1007/978-14614-1590-9_24

Christianson GB, Peña JL (2006) Noise reduction of coincidence detector output by the inferior colliculus of the barn owl. J Neurosci 26(22):5948-5954

Fischer BJ, Christianson GB, Peña JL (2008) Cross-correlation in the auditory coincidence detectors of owls. J Neurosci 28(32):8107-8115

Funabiki K, Ashida G, Konishi M (2011) Computation of interaural time difference in the owl's coincidence detector neurons. J Neurosci 31(43):15245-15256

Gerstner W, Kempter R, van Hemmen JL, Wagner H (1996) A neuronal learning rule for submillisecond temporal coding. Nature 383(6595):76-78. doi:10.1038/383076a0

Joris PX, Carney LH, Smith PH, Yin TCT (1994) Enhancement of neural synchronization in the anteroventral cochlear nucleus. I. Responses to tones at the characteristic frequency. J Neurophysiol 71(3): 1022-1036

Kempter R, Gerstner W, van Hemmen JL, Wagner H (1998) Extracting oscillations: Neuronal coincidence detection with noisy periodic spike input. Neural Comput 10(8):1987-2017

Köppl C (1997) Frequency tuning and spontaneous activity in the auditory nerve and cochlear nucleus magnocellularis of the barn owl Tyto alba. J Neurophysiol 77:364-377

Köppl C, Carr CE (2008) Maps of interaural time difference in the chicken's brainstem nucleus laminaris. Biol Cybern 98(6):541-559. doi:10.1007/s00422-008-0220-6

Kuokkanen PT, Ashida G, Carr CE, Wagner H, Kempter R (2013) Linear summation in the barn owl's brainstem underlies responses to interaural time differences. J Neurophysiol 110(1):117-130. doi: $10.1152 /$ jn. 00410.2012

Kuokkanen PT, Wagner H, Ashida G, Carr CE, Kempter R (2010) On the origin of the extracellular field potential in the nucleus laminaris of the barn owl (Tyto alba). J Neurophysiol 104(4):2274-2290. doi:10.1152/jn.00395.2010 
Mc Laughlin M, Verschooten E, Joris PX (2010) Oscillatory dipoles as a source of phase shifts in field potentials in the mammalian auditory brainstem. J Neurosci 30(40):13472-13487. doi:10.1523/JNEUROSCI.0294-10.2010

McColgan T, Shah S, Köppl C, Carr CE, Wagner H (2014) A functional circuit model of interaural time difference processing. J Neurophysiol 112(11):2850-2864. doi:10.1152/jn.00484.2014

Pena JL, Konishi M (2000) Cellular mechanisms for resolving phase ambiguity in the owl's inferior colliculus. Proc Natl Acad Sci U S A 97(22):11787-11792. doi:10.1073/pnas.97.22.11787

Recio A, Rich N, Narayan S, Ruggero M (1998). Basilar-membrane responses to clicks at the base of the chinchilla cochlea. J Acoust Soc Am 103(4):1972-1989.

Ruggero M, Robles L, Rich N, Costalupes J (1986) Basilar membrane motion and spike initiation in the cochlear nerve. In: Moore BCJ, Patterson RD (eds) Auditory frequency selectivity (119 ed., Vol. 189). Auditory Frequency Selectivity, London

Sachs M, Young E, Lewis R (1974) Discharge patterns of single fibers in the pigeon auditory nerve. Brain Res 70(3):431-447

Sanes DH, Constantine-Paton M (1985) The sharpening of frequency tuning curves requires patterned activity during development in the mouse, Mus musculus. J Neurosci 5(5):1152-1166

Seidl AH, Rubel EW, Harris DM (2010) Mechanisms for adjusting interaural time differences to achieve binaural coincidence detection. J Neurosci 30(1):70-80. doi:10.1523/JNEUROSCI.3464-09.2010

Sullivan WE, Konishi M (1986) Neural map of interaural phase difference in the owl's brainstem. Proc Natl Acad Sci U S A 83:8400-8404

Wagner H, Brill S, Kempter R, Carr CE (2005) Microsecond precision of phase delay in the auditory system of the barn owl. J Neurophysiol 94(2):1655-1658

Wagner H, Brill S, Kempter R, Carr CE (2009) Auditory responses in the barn owl's nucleus laminaris to clicks: impulse response and signal analysis of neurophonic potential. J Neurophysiol 102(2): 1227-1240. doi:10.1152/jn.00092.2009

Wagner H, Takahashi T, Konishi M (1987) Representation of interaural time difference in the central nucleus of the barn owl's inferior colliculus. J Neurosci 7(10):3105-3116

Young E, Robert J, Shofner W (1988) Regularity and latency of units in ventral cochlear nucleus: implications for unit classification and generation of response properties. J Neurophysiol 60(1):1-29 